\title{
El contenido estereotípico y el discurso acerca del prejuicio racial en Bahía
}

\author{
Stereotypic Content and the Discourse about \\ Racial Prejudice in Bahia \\ O conteúdo estereotípico e o discurso acerca \\ do preconceito racial na Bahia
}

\author{
Elza Maria Techio* \\ Universidad Federal de Bahía \\ Eldo Lima Leite** \\ Renata Pimentel da Silva** \\ Ana Raquel Rosas Torres** \\ Universidad Federal da Paraíba
}

Doi: http://dx.doi.org/10.12804/revistas.urosario.edu.co/apl/a.6158

\section{Resumen}

Esta investigación tuvo como objetivo descubrir el contenido de los estereotipos asociados a las personas racializadas blancas y negras, así como verificar las justificaciones para la existencia de prejuicio racial en Bahía. En esta encuesta participaron 146 personas, de las cuales un $56.8 \%$ fueron estudiantes de una universidad pública de Salvador de Bahía y un 43.2\% no estudiantes, con edades comprendidas entre 16 y 75 años $(\mathrm{M}=$ $29.84 \mathrm{DT}=12.663)$, el $47.6 \%$ eran de sexo femenino y el 52.4\% de sexo masculino. Los participantes respondieron a una prueba de asociación libre, que contenía las palabras estímulos: personas negras y blancas; además de una pregunta referente a la existencia de prejuicio en Bahía para justificar su respuesta. Los contenidos de los estereotipos indican que a las personas racializadas como blancas y a las negras se les asocian estereotipos referentes al estatus y condiciones sociales. Las personas blancas fueron percibidas como poseedores de un mayor estatus (ricos y guapos) y las personas negras con menor (pobres y trabajadores), lo que corroboró

* Universidad Federal de Bahía

** Universidad Federal da Paraíba

Contacto para correspondencia editorial: Elza Maria Techio Psicóloga pela Universidade Federal da Paraíba (1996), Licenciada em Psicologia pela ufpb (1994), mestre em Psicologia Social pela ufpb (1999), e doutora em Psicologia Social pela Universidad del Pais Vasco, Espanha (2008). Professora do programa de pós-graduação em Psicologia Social da Universidade Federal da Bahia. Tem experiência na área de Psicologia, com ênfase em Psicologia Social. Correo electrónico: elzamt400@gmail.com

Cómo citar este artículo: Techio, E. M., Leite, E. L., da Silva, R. P., \& Torres, A. R. R. (2019). El contenido estereotípico y el discurso acerca del prejuicio racial en Bahía. Avances es Psicología Americana, 37(1), 179-194. Doi: http://dx.doi.org/10.12804/ revistas.urosario.edu.co/apl/a.6158 


\section{Elza Maria Techio, Eldo Lima Leite, Renata Pimentel da Silua, Ana Raquel Rosas Torres}

el modelo del contenido de los estereotipos de Fiske et al. $(1999,2002,2007)$. Además, los participantes afirmaron que perciben la existencia del prejuicio racial en Bahía. Las justificaciones se agruparon en dos categorías. La primera presenta el prejuicio racial como un fenómeno histórico, mientras que en la segunda el prejuicio racial es visto como un fenómeno actual. Por lo tanto, se pudo verificar que el prejuicio racial fue percibido como un fenómeno social cuyas raíces están en la estructura social brasileña, que lo configura como un fenómeno institucional, que cuestiona el mito de la democracia racial en Brasil.

Palabras clave: contenido de los estereotipos, prejuicio racial, prejuicio institucional.

\section{fbstract}

This research aimed to investigate the content of stereotypes associated with racialized white and black people, and to verify the justifications for the existence of racial prejudice in Bahia. 146 people participated, of which 83 were university students and 63 were non-university students. Participants responded a free association task, containing the words stimuli: black and white people; and a question regarding the existence of prejudice in Bahia, justifying its response. The contents of stereotypes indicate that stereotypes regarding status and social conditions are associated with whites and blacks. White people were perceived to have higher status (rich and beautiful) and lower status black people (poor and workers), corroborating the stereotype content model of Fiske et al. (1999, 2002, 2007). The participants affirmed that there was racial prejudice in Bahia. The justifications were grouped into two clusters. The first show racial prejudice as a historical phenomenon, while in the second, prejudice is seen as a current phenomenon. Therefore, it can be verified that racial prejudice was perceived as a social phenomenon whose roots are in the Brazilian social structure, forming itself as an institutional phenomenon, questioning the myth of racial democracy in Brazil.

Keywords: Contents of stereotypes, racial prejudice, institutional prejudice.

\section{Resumo}

Esta pesquisa teve como objetivo descobrir o conteúdo dos estereótipos associados às pessoas racializadas brancas e negras, assim como verificar as justificações para a existência de preconceito racial na Bahia. Neste inquérito participaram 146 pessoas, das quais um $56.8 \%$ foram estudantes de uma universidade pública do Salvador da Bahia e um $43.2 \%$ não estudantes, com idades compreendidas entre 16 e 75 anos $(\mathrm{M}=29.84$ DT $=12.663$ ), sendo o $47.6 \%$ de sexo feminino e o $52.4 \%$ de sexo masculino. Os participantes responderam a uma tarefa de associação livre, contendo as palavras estímulos: pessoas negras e brancas; e uma pergunta referente à existência de preconceito na Bahia, justificando a sua resposta. Os conteúdos dos estereótipos indicam que às pessoas racializadas como brancas e às negras se lhes associam estereótipos referentes ao status e condições sociais. As pessoas brancas foram percebidas como possuidores de maior status (ricos e bonitos) e as pessoas negras com menores status (pobres e trabalhadores), corroborando o modelo do conteúdo dos estereótipos de Fisk et al. (1999, 2002, 2007). Além disso, os participantes afirmaram que percebem a existência do preconceito racial na Bahia. As justificações se agruparam em dois clusters. O primeiro presenta o preconceito racial como um fenômeno histórico, enquanto que no segundo o preconceito racial é visto como um fenômeno atual. Portanto, conseguiu-se verificar que o preconceito racial foi percebido como um fenômeno social cujas raízes estão na estrutura social brasileira, configurando-se como um fenômeno institucional, questionando o mito da democracia racial no Brasil.

Palavras-chave: conteúdo dos estereótipos, preconceito racial, preconceito institucional.

\section{Introducción}

La sociedad brasileña está marcada de forma negativa por la desigualdad social. De acuerdo con los datos del Instituto Brasileño de Geografía y Estadística (IBGE, 2014), entre el 1\% más rico de 
la población brasileña, un $83.6 \%$ se corresponde a las personas racializadas como blancas, mientras que solo un $14.6 \%$ son personas racializadas como negras o pardas ${ }^{1}$. En cuanto al $10 \%$ más pobre, el $75 \%$ son negros o pardos y el $23.9 \%$, blancos. A raíz de estos datos subyace la idea de que existe un favorecimiento socioeconómico por las personas racializadas como blancas sobre las personas racializadas como negras (Munanga, 2007). Esta desigualdad sería reflejo de los procesos históricos de explotación, exclusión social y prejuicio vividos por los negros en Brasil debido a la compleja estructuración social y racial derivada de la esclavitud y del desamparo del Estado que sufrió la población negra cuando esta se abolió (Batista, Leite, Camino \& Torres, 2014; Camino, da Silva, Machado \& Pereira, 2001).

En la historia de Brasil (Schwarcz \& Starling, 2015) y de la formación de la sociedad brasileña, la ciudad de Salvador de Bahía fue la que sufrió el mayor impacto del proceso de esclavitud. Salvador fue la puerta de entrada de los negros cautivos que llegaron al país durante la colonización. De acuerdo con los datos del IBGE (2014), Salvador es considerada la ciudad más negra de Brasil y la cuna de la cultura afrobrasileña (Moreno, 2016). Expresiones culturales que se mantienen hasta la actualidad en la musicalidad, la culinaria y las manifestaciones religiosas. Cerca del $79.47 \%$ de la población de Salvador se autodeclara como de color negro $(51.67 \%)$ y pardo $(27.80 \%)$.

Expresiones de discriminación contra las personas racializadas negras, sea mediante prácticas individuales o institucionales (individuo que actúa como representante de la institución) se observan en todas las regiones de Brasil. Aunque, Salvador de Bahía es la ciudad más negra de Brasil, expre-

Persona parda: terminología utilizada por el IBGE (2014) para configurar uno de los cinco "colores o raza" que componen la población brasileña: blanca, parda, negra, amarilla e indígena. Parda es una persona con mistura de color de piel (blanco con negro, blanco con indígena o negro con indígena). siones de prejuicio racial, racismo y discriminación también son observadas.

En Brasil, la población negra es la más afectada por la violencia y el desempleo: tiene los sueldos más bajos, menos acceso al sistema de salud, a la escuela y a la vivienda, son la más pobre y vulnerable socialmente (Paixão, Rossetto, Montovanele \& Carvano, 2010). Datos del mapa de la violencia (Waiselfisz, 2016) destacan que las personas racializadas negras son más víctimas de homicidios, las más encarceladas y las que más sufren violencia policial, sobre todo los jóvenes negros que viven en las periferias. Esta situación revela que las desigualdades sociales en Brasil tienen color y están ancladas en el racismo institucional, mediante políticas públicas que tratan a la población negra y pobre de forma diferenciada.

Resumiendo, por ser Salvador de Bahía la ciudad más negra de Brasil y por ser la población negra una de las más expuestas al prejuicio racial en un país que se ve como una democracia racial (Freyre, 1933/1998), esta investigación tuvo como objetivo investigar el contenido de los estereotipos asociados a las personas racializadas como blancas y negras, así como verificar las justificaciones para la existencia del prejuicio racial en Bahía.

El prejuicio como problema social pasó a recibir mayor énfasis en la psicología social después de la primera publicación en 1954 del libro La Naturaleza del prejuicio de Gordon W. Allport. Este autor definió prejuicio como "una antipatía basada en una generalización equivocada e inflexible. Puede ser sentido o expresado. Se dirige a un grupo como un todo, o a un individuo por formar parte de este grupo" (1979, p. 9). Para Brown (2010), el prejuicio además de evaluaciones y creencias negativas sobre los grupos incluye prácticas discriminatorias dirigidas a estos. La discriminación se refiere al aspecto conductual del prejuicio. Se trata de un comportamiento diferenciado y dirigido a individuos o grupos de determinadas categorías sociales (Brewer, 1994). Son acciones que crean o preservan ventajas de un grupo sobre otro, cuyo 
principal objetivo sería legitimar las desigualdades sociales (Jones, 1997; Schucman, 2014).

En Brasil, diversos estudios han demostrado que a pesar de la propagación del mito de la democracia racial, la sociedad brasileña percibe que en la actualidad aún existe prejuicio racial. Sin embargo, es generalmente percibido como responsabilidad de la sociedad como un todo y no del propio individuo perceptor (Batista et al., 2014; Camino et al., 2001). Estos resultados parecen ser reflejo del efecto de las normas sociales, que prohíben expresiones abiertas de prejuicio racial, lo que llevaría a manifestaciones más sutiles de este (Bergsieker \& Leslie, 2012; Pereira, Torres \& Almeida, 2003; Pereira \& Vala, 2010). Debido a los cambios en la expresión de dicho fenómeno, los investigadores sociales desarrollaron teorías que pudieron abordar las nuevas formas del prejuicio y del racismo (Camino et al., 2001; Gaertner \& Dovidio, 1986; Katz \& Hass, 1988; Kinder \& Sears, 1981; Pedersen \& Walker, 1997; Pettigrew \& Meertens, 1995; Turra \& Venturi, 1995).

Este conjunto de teorías sugiere que el prejuicio es un fenómeno difícil de abordar dada su maleabilidad social (Unnever \& Cullen, 2012). Uno de los problemas de abarcar el prejuicio como un todo es que este no es un fenómeno unidimensional. El prejuicio, concebido como una evaluación o una actitud, se compondría de tres componentes: el cognitivo, que hace referencia a los estereotipos: creencias sobre las características de un determinado grupo social; el afectivo, que remite a las sensaciones y emociones asociadas a los grupos sociales, y el disposicional, referente al comportamiento de discriminar a individuos o grupos (Techio, 2011).

Para Allport (1979), el aspecto cognitivo del prejuicio, formado por los estereotipos y creencias sobre los grupos, explica en parte la inflexibilidad de las actitudes prejuiciosas. Esto se debe a que existe una tendencia en los individuos a percibir el mundo en categorías. En este sentido, el proceso de estereotipia sería un producto normal de los procesos de categorización (Techio, 2011) y tendría una estrecha relación con el prejuicio (Amodio \& Devine, 2006; Crandall, Bahns, Warner \& Schaller, 2011; Gordijn, Koomen \& Stapel, 2001; Fehr, Sassenberg \& Jonas, 2012; Sparkman \& Eidelman, 2016).

Los estereotipos no son solo un aspecto del prejuicio, también son parte fundamental del funcionamiento psíquico. Entre las principales funciones de los estereotipos se encuentran la conservación de los recursos mentales (Fiske \& Taylor, 1991), defensa de los valores individuales (Guan, Deng \& Bond, 2010), explicación y búsqueda de causalidad social (Sherman, Stroessner, Conrey \& Azam, 2005), diferenciación social (Gawronski, Deutsch, Mbirkou, Seibt \& Strack, 2008) y justificación social (Batista et al., 2014; Camino et al., 2001; Lima \& Vala, 2004; Techio, 2011).

Pero si los estereotipos se asocian a los grupos (Tajfel \& Turner, 1979), ¿cuál es la dimensión de los estereotipos que caracteriza al endogrupo que apoya para establecer la diferencia con el exogrupo? Las características atribuidas a los grupos pueden ser tanto positivas como negativas, pueden ser correctas o equivocadas (Techio, 2011). Esto nos lleva a creer que, respecto al proceso de estereotipia, son los contenidos que componen el significado de la categoría social lo que nos lleva a diferenciar a los grupos. Podemos afirmar entonces que los estudios sobre los estereotipos pueden ser clasificados en dos grandes grupos. Los que investigan los procesos involucrados en su construcción y los que enfatizan los contenidos que los forman.

Los estudios que tienen como foco los procesos cognitivos tienen como objetivo comprender cómo los estereotipos se forman, y cuál es su origen, sus procesos y sus funciones (Oakes, Haslan \& Turner, 1994). El enfoque que defiende que los procesos de estereotipar responden a principios sistemáticos pueden ser generalizados y se muestran estables a lo largo del tiempo y en diferentes lugares (Fiske, Cuddy, Glick \& Xu, 2002). Los estereotipos como 
estructuras de conocimiento estarían articulados con otros procesos cognitivos con la intención de hacer frente a las demandas de la realidad social buscando simplificarla y ordenarla y, al mismo tiempo, interfiriendo en procesos como percepción, asignación de atención, registro de informaciones y en la evocación de contenidos de memorias (Pereira, 2015).

Los primeros estudios de mayor impacto sobre el contenido de los estereotipos fueron desarrollados en la universidad de Princeton, por Katz y Braly (1933), Gilbert (1951) y Karlins, Coffman y Walters (1969). Esta serie de estudios demostró que los adjetivos utilizados por los estudiantes a lo largo de 37 años permanecieron relativamente estables. En los estudios de Gilbert (1951) y Karlins et al., (1969), los estudiantes usaron más adjetivos para caracterizar cada grupo, en comparación con el estudio de Katz y Braly (1933). No obstante, el contenido permaneció similar. Estos estudios se conocieron como la Trilogía de Princeton.

Para Fiske, Xu, Cuddy y Glick (1999), el contenido de los estereotipos puede clasificarse en dos dimensiones: competencia y sociabilidad. Para estos autores los estereotipos surgen a partir de la estructura de las relaciones intergrupales como consecuencia del grupo ser percibido con alto o bajo estatus y por el nivel de interdependencia si el grupo es visto como cooperativo o competitivo. Así, el estatus funcionaría como predictor de la competencia y la interdependencia como predictor de la sociabilidad (Fiske et al., 2002; Fiske, Cuddy \& Glick, 2007; Fiske et al., 1999). Además de estos estudios, una serie de trabajos han demostrado la validez de esta estructura bidimensional de los estereotipos (Batista et al., 2014; Bergsieker \& Leslie, 2012; Binggeli, Krings \& Sczesny, 2014; Crandall, et al., 2011; Guan et al., 2010; Sadler, Meagor \& Kaye, 2012).

A partir de estas consideraciones, este trabajo tuvo como objetivo averiguar el contenido de los estereotipos asociados a las personas racializadas como blancas y a las racializadas como negras, además de verificar los repertorios discursivos de las justificaciones para la existencia de prejuicio racial en Bahía, estado brasileño con mayor número de afrodescendientes.

\section{Método}

\section{Participantes}

Participaron en la investigación 146 personas, un $56.8 \%$ fueron estudiantes de una universidad pública de Salvador de Bahía y un $43.2 \%$ no estudiantes, con edades entre 16 y 75 años $(\mathrm{M}=29.84$ $\mathrm{DT}=12.663$ ), el $52.4 \%$ fueron hombres y $47.6 \%$ fueron mujeres. La renta media familiar fue de $\$ 3590.99$ reales $(\mathrm{DT}=3789.19)$. El color de la piel autodeclarada, utilizando la clasificación del IBGE, fue: $39.2 \%$ como pardo (morenos y mulatos), un $25.2 \%$ se definió como negro, un $24.5 \%$ como blanco y un $11.2 \%$ como otros (indígenas, amarillos y mestizos). El sistema de clasificación de color y raza del IBGE (2014) sugiere que las personas negras y pardas pertenecen a la misma categoría racial; o sea, son clasificadas como personas negras.

\section{Procedimientos e instrumentos}

La muestra fue no probabilística por conveniencia. Los estudiantes universitarios respondieron al instrumento individualmente en los diversos espacios físicos de la universidad (aulas, pasillos de la universidad, biblioteca, etc.), y los no universitarios respondieron individualmente al cuestionario en sus casas, plazas, centros comerciales, etc. Los participantes mediante la evocación de palabras debían recordar las características (estereotipos) atribuidas a las personas racializadas como blancas y negras. En seguida respondieron a una escala de cuatro puntos que evalúa la percepción de la existencia de prejuicio racial en Bahía (1 mucho, 2 más o menos, 3 poco y 4 nada). Para el análisis de los datos, los valores de la escala fueron in- 
vertidos y dicotomizados, resultando 1 para "no existe prejuicio" y 2 "existe prejuicio en Bahía". Los participantes también debieron justificar su respuesta. Finalmente, respondieron a un cuestionario con preguntas sobre sus características sociodemográficas.

\section{Aspectos éticos}

Todos los procedimientos éticos prescritos en la Resolución 466/2012 del Consejo Nacional de Salud (CNS) fueron seguidos y los términos de consentimiento libre y aclarado fueron debidamente firmados por los participantes.

\section{Análisis de datos}

Las respuestas de las evocaciones se analizaron mediante el programa EVOC 2003, que trata de combinar la frecuencia con que se recuerdan las palabras y el orden de evocación. Este programa permite aprehender los elementos centrales y periféricos presentes en el discurso de los sujetos, que configuran las representaciones sociales.

El análisis de la cuestión abierta se realizó por medio del software Iramuteq (Interface de R pour les Analyses Multidimensionnelles de Textes et de Questionnaires) desarrollado por Pierre Ratinaud (2009). Este software permite a los investigadores utilizar diferentes recursos técnicos de análisis léxico. En los análisis léxicos clásicos, el software identifica los textos, los transforma en segmentos de textos, realiza la investigación del vocabulario y reduce las palabras con base en sus raíces, y crea formas reducidas y complementarias (Camargo \& Justo, 2013). Los demás datos se analizaron a través del programa SPSS.

\section{Resultados}

El análisis de los datos mediante el uso del EVOC indicó la ocurrencia de 328 palabras evocadas para el término inductor "personas negras",
180 palabras fueron distintas, aparecen solo una vez. Para el término inductor "personas blancas", se obtuvieron 321 palabras, de las que 168 fueron distintas. Se adoptó como punto de corte para el orden medio 2.5 (enumeradas de 1 a 5 , en las que 1 era la más importante) y límites de frecuencia entre 4 y 10 . En la tabla 1 se representan los principales elementos evocados.

Para el término inductor "personas negras", el programa identificó 15 palabras más frecuentes: pobres, trabajadores, fuertes, guapos, cabello rizado, negrazo, piel, resistencia, burros, cabellos, feos, humildes, ladrones, perezosos y sensuales. El software también identificó la coocurrencia entre las palabras evocadas, que pueden estar relacionadas o pertenecer a la misma clase. La palabra pobre(s) presenta una fuerte coocurrencia con la palabra ladrón(es), una vez que aparece siete veces acompañada de esta y reiterando el sentido de esta asociación aparece a veces acompañada de las palabras criminales y bandidos. La palabra piel se evocó tres veces junto con la expresión de cabello rizado.

Para el término inductor "personas blancas", el programa identificó 20 palabras más frecuentes: educados, feos, limpios, opresores, piel, prejuicio, trabajadores, guapos, ricos, inteligentes, belleza, blanquitos, cabello, élite, inteligencia, lacio, rubios, racistas y superiores. La mayor coocurrencia fue entre las palabras hermoso y limpio, evocadas juntas 6 veces, así como bonito y rico evocadas 4 veces juntas. Además, en la clase "personas negras" la palabra piel también fue evocada junto a la expresión referente al cabello, que en esta categoría la expresión fue cabello lacio. Lo que sugiere que el tipo de cabello (lacio y rizado) es una característica importante para cualificar la categoría social de pertenencia.

Conforme a la tabla 1 , el contenido central de los estereotipos atribuidos a las personas negras, representados en el primer cuadrante, implica elementos asociados a la condición de ser pobres y trabajadores, mientras que los elementos asociados a 
las personas blancas hacen referencia a la condición de ser bonitos y ricos. Para ambas, las categorías raciales blancos y negros se activan características estereotípicas asociadas a las condiciones sociales y estatus ocupadas por estas en la sociedad brasileña. Las personas racializadas como blancas son reconocidas como poseedoras de un mayor estatus (ricos y bonitos) y las personas racializadas como negras menor estatus (pobres y trabajadores). En cierta forma, los elementos evocados diferencian a los grupos y las características más positivas son atribuidas a las personas blancas y las negativas a las personas negras. Según Abric (2003), los elementos contenidos en el primer cuadrante, los primeros y más frecuentes a ser evocados, son considerados centrales y representan los elementos más estables en la representación social.

En la primera periferia se encuentran los elementos que presentan alta frecuencia y baja importancia, que complementan el núcleo central y dan sentido y significado a las representaciones, así como influencian las prácticas sociales. En este sentido, la característica atribuida a las personas negras fue fuerza, que está asociada a la habilidad y fuerza física; mientras que a las personas blancas fue inteligencia, característica asociada a la intelectualidad.

Entre las características presentes en la zona de contraste (tercer cuadrante) se encuentran los estereotipos asociados a las personas negras como: guapos, cabello rizado, negrazo, piel y resistencia; así como los asociados a las personas blancas: belleza, blanquitos, cabello, élite, inteligencia, lacio, rubios, racistas y superiores. Estos elementos presentan baja frecuencia y alta importancia y se caracterizan por su fluidez y mayor posibilidad de cambio. Para Abric (2003) y Rocha (2010), los elementos presentes en este cuadrante pueden expresar tanto un subtipo de representación como ser un elemento complementario de la primera periferia o incluso pueden indicar cambios en la representación, en este caso, un cambio en el contenido de los estereotipos. La enunciación de características fenotípicas positivas asociadas a las personas negras como bonitos, que sugiere el tipo de cabello rizado y el de piel: negrazo, puede indicar la existencia de un subgrupo de personas que valora las características fenotípicas de las personas negras, mientras que el adjetivo "resistencia" refuerza la representación del papel social de las personas negras para el trabajo físico o expresar la resistencia y lucha por el reconocimiento social y fortalecimiento de su identidad. Mientras que los estereotipos atribuidos en este cuadrante parecen reforzar la representación central de que las personas blancas son superiores, inteligentes, bonitas, en especial, al asociar el tipo de cabello (liso y rubio) y el color de la piel, también surgen características negativas asociadas al hecho de ser percibidas como racistas. De todas formas, se mantiene intacta la creencia de superioridad, mayor estatus social y competencia de las personas racializadas como blancas sobre las racializadas como negras.

Finalmente, en la segunda periferia se encuentran los elementos con baja frecuencia y baja importancia. Los elementos que forman parte del sistema periférico de las representaciones y que se consideran más emancipados mantienen cierto grado de independencia de la representación central, que es más hegemónica (Cabecinhas, 2004), son valores y posicionamientos individuales que están en constante transformación (Carvalho, Lima, Faro \& Silva, 2012) y vinculados a las experiencias inmediatas y asociadas a las prácticas sociales (Techio, Gonçalves \& Costa, 2016). Las evocaciones de burros, cabello, feos, humildes, ladrones, perezosos y sensuales atribuidos a las personas negras acaban por reforzar la visión negativa que se tiene de la categoría racial negros en la sociedad brasileña, en especial en Bahía. Mientras que las evocaciones atribuidas a la categoría racial blancos presentan un carácter más ambivalente, mezclando características positivas (educados, limpios y trabajadores) y negativas (feos, opresores y prejuiciosos). 
Tabla 1.

Análisis estructural de los estereotipos atribuidos a los negros y blancos

\begin{tabular}{|c|c|c|c|c|c|}
\hline \multicolumn{6}{|c|}{$\begin{array}{c}\text { Núcleo central } \\
\text { Frecuencia } \geq 10 \text { y Clasificación media } \leq 2.5\end{array}$} \\
\hline \multicolumn{3}{|c|}{ Negros } & \multicolumn{3}{|c|}{ Blancos } \\
\hline Evocación & Frec $^{1}$ & Orden $^{2}$ & Evocación & Frec $^{1}$ & Orden $^{2}$ \\
\hline Pobres & 24 & 2.21 & Bonitos & 17 & 2.35 \\
\hline Trabajadores & 10 & 2.20 & Ricos & 25 & 2.40 \\
\hline \multicolumn{6}{|c|}{$1^{\text {a }}$ Periferia } \\
\hline \multicolumn{3}{|c|}{ Negros } & \multicolumn{3}{|c|}{ Blancos } \\
\hline Evocación & Frec $^{1}$ & Orden $^{2}$ & Evocación & Frec $^{1}$ & Orden $^{2}$ \\
\hline Fuertes & 13 & 2.54 & Inteligentes & 14 & 2.57 \\
\hline \multicolumn{6}{|c|}{$\begin{array}{l}\text { Zona de contraste } \\
\text { Frecuencia } \geq 9 \text { y Clasificación media } \leq 2,5\end{array}$} \\
\hline \multicolumn{3}{|c|}{ Negros } & \multicolumn{3}{|c|}{ Blancos } \\
\hline Evocación & Frec $^{1}$ & Orden $^{2}$ & Evocación & Frec $^{1}$ & Orden $^{2}$ \\
\hline Bonitos & 7 & 2.43 & Belleza & 5 & 2.20 \\
\hline Rizado & 6 & 2.33 & Blanquitos & 5 & 2.00 \\
\hline Negrazo & 4 & 2.00 & Cabello & 9 & 2.33 \\
\hline Piel & 6 & 2.17 & Elite & 4 & 2.12 \\
\hline \multirow[t]{5}{*}{ Resistencia } & 4 & 2.00 & Inteligencia & 4 & 2.00 \\
\hline & & & Lacio & 7 & 2.29 \\
\hline & & & Rubio & 4 & 2.00 \\
\hline & & & Racistas & 5 & 2.00 \\
\hline & & & Superiores & 4 & 2.25 \\
\hline \multicolumn{6}{|c|}{$2^{\text {a }}$ Periferia } \\
\hline \multicolumn{3}{|c|}{ Negros } & \multicolumn{3}{|c|}{ Blancos } \\
\hline Evocación & Frec $^{1}$ & Orden $^{2}$ & Evocación & Frec $^{1}$ & Orden $^{2}$ \\
\hline Burros & 7 & 2.80 & Educados & 7 & 2.71 \\
\hline Cabello & 9 & 2.56 & Feos & 4 & 3.01 \\
\hline Feos & 8 & 2.87 & Limpios & 4 & 2.50 \\
\hline Humildes & 5 & 2.80 & Opresores & 6 & 2.83 \\
\hline Ladrones & 4 & 3.00 & Piel & 4 & 2.50 \\
\hline Perezosos & 7 & 2.57 & Prejuiciosos & 6 & 2.83 \\
\hline Sensuales & 4 & 2.50 & $\begin{array}{l}\text { Trabajado- } \\
\text { res }\end{array}$ & 4 & 2.75 \\
\hline
\end{tabular}

Nota: Frec ${ }^{l}$ Frecuencia absoluta; Orden ${ }^{2}$ Orden de clasificación
Después del análisis del núcleo central y periférico de la representación de los rasgos estereotípicos atribuidos a las personas pertenecientes a las categorías raciales blancos y negros, se realizaron una seria de análisis de variancia para verificar si el sexo, el color de la piel de los participantes y el hecho de ser un estudiante universitario o no, afectaban la percepción de la existencia de prejuicio racial en Bahía. Los resultados no indicaron diferencias estadísticas significativas cuando se comparan las respuestas de los hombres $(\mathrm{M}=3.64 ; \mathrm{DT}=0.626)$ y las mujeres $(\mathrm{M}=$ $3.72 ; \mathrm{DT}=0.482), \mathrm{F}(1 ; 144)=0.731 ; \mathrm{p}<0.394$; de las personas que se autodeclararon blancas $(\mathrm{M}=3,60 ; \mathrm{DT}=0.604)$, negras $(\mathrm{M}=3.78$; $\mathrm{DT}=0.485)$, pardas $(\mathrm{M}=3.66 ; \mathrm{DT}=0.611) \mathrm{y}$ otras $(\mathrm{M}=3.75$; $\mathrm{DT}=0.447), \mathrm{F}(3.142)=0.696$; $\mathrm{p}<0.556$, ni de los estudiantes $(\mathrm{M}=3.69$; $\mathrm{DT}=0.582)$ y no estudiantes $(\mathrm{M}=3.68$; $\mathrm{DT}=0.534), \mathrm{F}(1 ; 144)=0.002 ; \mathrm{p}<0.964$.

Al mismo tiempo, los análisis indican la percepción que existe prejuicio racial en Bahía $(\mathrm{M}=3.68$, $\mathrm{DT}=0.56)$. Estos resultados están en consonancia con estudios sobre la percepción del racismo en Brasil (Batista et al., 2014, Camino et al., 2001) y en Río de Janeiro (Oliveira \& Barreto, 2003). Con la intención de esclarecer tal resultado, se pasó a analizar el discurso de los participantes de la investigación sobre los motivos o justificaciones sobre la existencia del prejuicio racial en Bahía.

El análisis léxico de la pregunta “¿En su opinión existe prejuicio racial en Bahía? Justifique su respuesta" presentó un total de 2836 ocurrencias con 914 formas distintas. El corpus fue compuesto por un número de textos igual a 134 , repartidos en 136 unidades de segmento de texto. Las unidades de segmentos de texto se dividieron en dos grupos, que se subdividieron en seis clases distintas, que representan el conjunto de los discursos pronunciados por los participantes. La presentación se hará con base en los dos grupos demostrados en el dendograma (figura 1) para posibilitar una mejor comprensión del análisis. 
El primero, denominado "Prejuicio actual", retrata los aspectos institucionales del prejuicio y abarca las clases: prejuicio institucionalizado, prejuicio y discriminación y prejuicio velado.

La clase prejuicio institucionalizado presenta el discurso que existe una discriminación recurrente en los espacios sociales e institucionales en la forma de tratamiento desigual atribuido a las personas blancas y negras; esta diferencia de trato no sería consecuencia solo de actitudes y creencias personales, sino de la estructura social brasileña en la que las personas blancas ocupan los mejores puestos de trabajo y de alto estatus social y las personas negras los peores puestos de trabajo y de bajo estatus: clara percepción de jerarquía social. Los discursos más representativos de esta clase fueron:

Empezando por los puestos de trabajo. Mientras el blanco alcanza puestos altos, los negros se quedan como conserjes domésticos. No se quiere que sobresalgan [...] Aunque vivimos en un Estado “negro", los valores y la posición social atribuidos a negros $\mathrm{o} / \mathrm{y}$ blancos son diversos y jerárquicamente diversos (sic). Las posibilidades también son diversas.

La clase denominada prejuicio y discriminación aparece asociada a la clase prejuicio institucionalizado y presenta la idea de que el prejuicio existe, y es expresado por medio de chistes con contenidos racistas, así como en la división social del trabajo, en la que las personas blancas son las que tienen más recursos financieros, son más competitivos y competentes y ocupan cargos caracterizados por el uso del intelecto. Las personas negras tienen menos recursos financieros y ocupan cargos caracterizados por el uso de la fuerza física y de menor estatus. Un segundo aspecto de esta clase es la ocurrencia de discursos que subrayan el lugar en la ciudad destinado a la población negra. Los discursos más representativos de esta fueron:

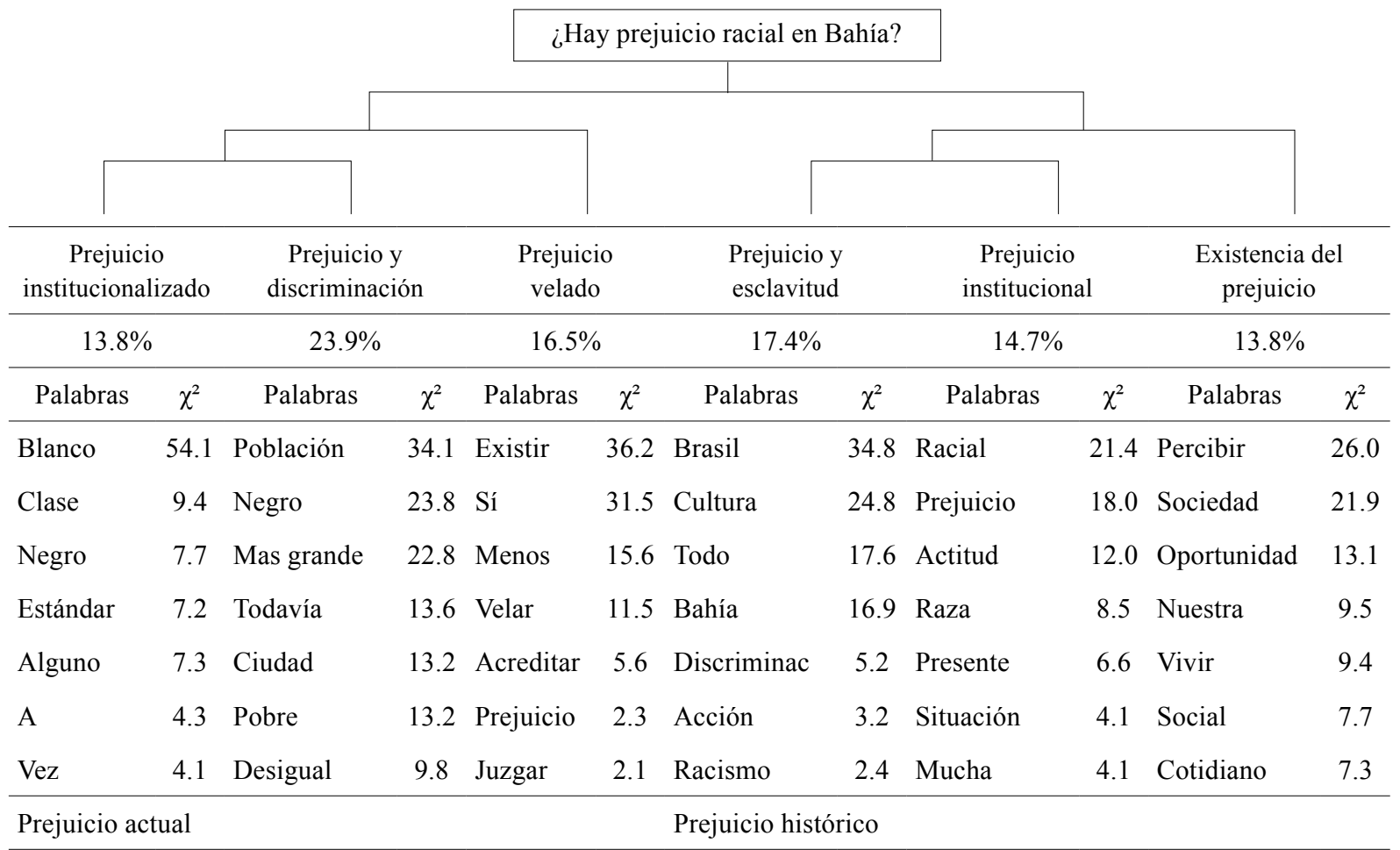

Figura 1. Dendograma de los discursos sobre la existencia de prejuicio en Bahía 


\section{Elza Maria Techio, Eldo Lima Leite, Renata Pimentel da Silua, fna Raquel Rosas Torres}

vemos el prejuicio en toda ciudad, donde edificios lujosos se construyen en barrios simples y demuestra la diferencia de clases sociales que existen, lo que involucra también la cuestión racial en discusiones normalmente en Bahía, la gente habla: "váyase negro" [...] En el acceso a los espacios públicos y privados, en el acceso a los empleos y puestos de poder, en el acceso a la calidad de vida y la dignidad de manera general.

La clase prejuicio velado presentó un discurso de que el prejuicio existe en la sociedad brasileña. Sin embargo, este no es manifestado abierta, sino veladamente. Los discursos más representativos de esta clase fueron: "dentro del Mito de la Democracia Racial se entiende que el negro es discriminado de forma perversa y disfrazada"; "Hay mucho prejuicio, sí, en Bahía y es de los peores, pues no es reconocido abiertamente".

El segundo grupo, prejuicio histórico, también vislumbra tres clases: prejuicio y esclavitud, prejuicio institucional y existencia del prejuicio. Este también confirma la percepción de la existencia del prejuicio contra las personas negras en Brasil y demuestra la presencia de aspectos históricos proveniente de la colonización y de la esclavitud que destacan las consecuencias del proceso de institucionalización del prejuicio.

La clase prejuicio y esclavitud presenta la idea de que el prejuicio existe, pero no es admitido. De acuerdo con las ideas contenidas en esta clase, el prejuicio sería una de las consecuencias del mito de la democracia racial y de la herencia esclavista sufrida por los negros. Los discursos más representativos de esta clase fueron: "el racismo es estructural en Brasil y Bahía tiene un historial de esclavitud mentalmente coronelista y racista"; "Porque esto está inserto en la cultura, aunque existe un intento de camuflaje".

La clase prejuicio institucional presenta el discurso de que el prejuicio sería un fenómeno institucional y nacional, que forma parte de la estruc- tura social brasileña, así como de su cultura. Los discursos más representativos de esta clase fueron

El prejuicio aparece muchas veces como algo interno. Hay una falsa idea de democracia racial que impidió la reflexión sobre el tema [...] En realidad, el prejuicio es algo muy presente en todo el país. Estamos acostumbrados a estereotipar a las personas por su condición racial.

Por último, la clase existencia del prejuicio reitera el discurso que el fenómeno del prejuicio existe en todo Brasil y es algo cotidiano. Los discursos más representativos de esta clase fueron:

A pesar de ser a menudo camuflado es posible percibir una discriminación inherente en nuestra sociedad [...] El prejuicio está impregnado en las relaciones sociales, en las empresas, en el acceso a las universidades, los salarios son diferenciados, las condiciones de vida y oportunidades, además de la imagen negativa.

\section{Discusiones}

El objetivo del presente trabajo fue reflexionar sobre el contenido de los estereotipos asociados a las personas racializadas como blancas y a las racializadas como negras, además de verificar la percepción de existencia de prejuicio racial en Bahía y los repertorios discursivos de las justificaciones para la existencia de prejuicio racial en Bahía.

En los resultados encontrados sobre el contenido de los estereotipos se percibe que a las personas racializadas como blancas se les atribuyen en general aspectos positivos que refuerzan la idea que es una categoría racial superior a la de los negros y que pertenece al grupo de alto estatus: ricos e inteligentes, asociados a características fenotípicas que son socialmente valoradas como cabello lacio y rubio. Incluso en la zona de contraste, el 
estereotipo atribuido a las personas blancas permanece vinculado al reconocimiento de superioridad racial, que indica consonancia entre los elementos periféricos y el núcleo central. En cambio, a las personas racializadas como negras se le atribuye pobreza y aptitud para el trabajo, reforzados por la primera periferia, en la que se evoca el término "fuertes", característica necesaria para el trabajo que requiere fuerza física. Los demás elementos de las periferias traen los estereotipos negativos que son recurrentes en la sociedad brasileña y atribuidos a las personas racializadas como negras, por ejemplo, ladrones y feos. Solo en la zona de contraste es donde la valorización de las características fenotípicas atribuidas a las personas racializadas como negras surge lo que puede indicar la existencia de un subgrupo minoritario que valora tales características, emergen cambios en los estereotipos atribuidos a la categoría racial negra.

Estos cambios pueden estar vinculados a la lucha de los diversos movimientos sociales negros que han reforzado y valorado las características culturales y fenotípicas de las personas racializadas como negras, que a su vez están asociadas al fortalecimiento de la identidad racial y la transformación de la percepción que se tiene de esta categoría social y racial (Carvalho \& Rocha, 2016; Gomes Lino, 2002). Lo que ocurre es que las mencionadas características negativas y socialmente desvalorizadas son reevaluadas para hacerlas positivas. Este fenómeno sucede, según Tajfel (1981), como una solución al hecho de que el grupo minoritario sea visto como diferente, en consecuencia, se busca modificar los aspectos que hacen destacadas tales diferencias.

Los elementos estereotípicos asociados a la población racializada como negra o como blanca, que se encuentran en la primera periferia, representan una visión basada en la dimensión de competencia y en al estatus social de Fiske et al. (2002): el rasgo inteligente asociado a la persona blanca remite al alto estatus, por consiguiente, a la alta competencia; mientras que el adjetivo fuerte asociado a la persona negra remite al bajo estatus y consecuentemente a la baja competencia. Estos elementos refuerzan y justifican la idea de que en la jerarquía social, la categoría social negro y los miembros pertenecientes a esa categoría son poco valorados socialmente. Resultados análogos a los encontrados en estudios anteriores. Batista et al. (2014), al analizar los adjetivos más representativos de las personas negras, encontraron que a la población negra se le asoció las características de trabajador y batallador/luchador, que según algunos autores, tales adjetivos pueden servir para reconocer la posición de inferioridad de estas personas.

Cabe destacar que los estereotipos aquí enumerados son compartidos independientemente del sexo, del color de la piel autoreportada y de la actividad realizada (estudiante / no estudiante). Esto confirma la hegemonía de los estereotipos en cuanto a creencias socialmente construidas y compartidas acerca de una categoría social, que interfieren en las relaciones que se establecen entre los grupos e influencian las actitudes y comportamientos intergrupales (Techio, 2011; Krüger, 2004; Lima \& Vala, 2004). La percepción e imagen negativa que se tiene de las personas negras como pertenecientes a categorías de menor prestigio y poco valoradas tales como: burros, feos, ladrones y perezosos, favorece y ayuda a justificar la existencia y mantenimiento del prejuicio y discriminación contra los negros en la sociedad brasileña fruto del proceso de socialización racista ocurrido a lo largo de la historia de Brasil, en consonancia con la existencia de normas sociales antiracistas (Batista et al., 2014, Camino et al., 2001, Lima \& Vala, 2004).

Existe un reconociendo de la existencia del prejuicio racial y racismo en la sociedad brasileña y en Salvador de Bahía, en especial cuando la población negra es percibida de forma negativa, mediante atribución de rasgos estereotípicos negativos. Los discursos acerca de la existencia del prejuicio confirman esta realidad de discriminación experimentada por las personas negras. 
La clasificación jerárquica descendiente (CHD) presentó un corpus dividido en dos grupos. El primero, prejuicio histórico, presenta el prejuicio como un fenómeno institucional e intrínseco a la estructura social brasileña. Este es considerado como una consecuencia del proceso de esclavitud vivido por los negros y por la creencia de que todas las razas en Brasil conviven de forma amistosa y respetuosa. El segundo, prejuicio actual, considera el prejuicio como una consecuencia de la propia estructura social, aunque se expresa de manera velada. También queda claro el proceso de discriminación demostrado en el papel social atribuido a cada grupo: a las personas blancas están reservados los mejores puestos de trabajo, de nivel intelectual, de alto estatus y bien pagados; a las personas negras los peores puestos de trabajo, que requieren fuerza física, bajos sueldo y de bajo estatus, resultados que corroboran el contenido de los estereotipos atribuidos a las personas racializadas como blancas y negras. Lo que se refleja en los elevados índices de desigualdad social y económica observados en Brasil (Relatório Anual das Desigualdades Raciais no Brasil, 2011).

Estos resultados corroboran los datos encontrados por Camino et al. (2013a) en cuanto a la conciencia de los participantes en la construcción sociohistórica del prejuicio y de la discriminación racial, que repercuten en la desigualdad percibida en las diferentes oportunidades de educación, así como en los diferentes roles sociales atribuidos a los negros, que repercuten en las oportunidades en el mercado de trabajo, que perpetúan las desigualdades sociales.

La idea que organiza los dos grupos es que el prejuicio sería un fenómeno institucional. De hecho, los discursos presentes en ambos hacen referencias al prejuicio como consecuencia de la estructura político-social brasileña, así como de la de su cultura. Para Werneck (2016), el racismo institucional se constituye como la dimensión más despreciada del prejuicio, que está asociada a una dimensión estructural relativa a las formas organizativas, políticas, prácticas y normas, que determinan los roles sociales desempeñados por los diferentes grupos sociales. Una de las principales consecuencias de este tipo de prejuicios sería la reproducción de la jerarquía social, que favorece a los grupos dominantes, para legitimar y justificar las diferencias sociales (Camino, Gouveia, Maia, Paz \& Laureano, 2013b, Lima \& Vala, 2004).

\section{Consideraciones finales}

El estudio del contenido de los estereotipos y del prejuicio en Brasil es importante para demostrar que las diferencias sociales, reales y percibidas entre la población blanca y negra, presentes en las representaciones y en el imaginario social en la sociedad brasileña son consecuencia de un proceso histórico de exclusión social que tiene como base el prejuicio racial y el racismo institucional. A partir del estudio aquí presentado, se pudo verificar que el prejuicio racial es percibido no solo como un simple fenómeno social, sino que también tiene sus raíces en la estructura social brasileña, configurándose como un fenómeno institucional (Werneck, 2016). Además, el presente estudio destaca que los procesos cognitivos de categorización social y el contenido de los estereotipos son fundamentales para entender el papel justificado de la discriminación social y racial. A partir del contenido de los estereotipos atribuidos a las personas negras y blancas, se pudo percibir que están asociados a diferentes papeles sociales, de modo que el grupo de los blancos fue más asociado a papeles de carácter intelectual, mientras que el grupo de los negros fue más asociado a papeles de carácter físico e instrumental. Estas imágenes serían derivadas de una construcción sociohistórica vinculada a la idea de la esclavitud sufrida por el grupo de los negros y del modo en que se establecieron las relaciones de poder derivadas de ese periodo.

Pensar el contenido de los estereotipos como derivados de una estructura social vigente desplaza 
la cuestión del cambio de los estereotipos de un nivel cognitivo hacia una discusión a nivel social. Este punto de vista establece una jerarquía de niveles de análisis, como propone Doise (1986), que posibilita diferentes maneras de abordar y operacionalizar este fenómeno. Una vez que los estereotipos asociados a las personas negras en Brasil están fundamentados no solo en características fenotípicas, sino también en la estructura social, nos encontramos con el problema de la retroalimentación del contenido de los estereotipos. Aunque se discute que los estereotipos atribuidos a los miembros de la categoría social negros son equivocados e imprecisos, ellos permanecen enraizados en la cultura y en las estructuras sociales, que demuestran que tales estereotipos son "ciertos" o al menos no son tan imprecisos. Si la fuente de los contenidos de los estereotipos es institucional, estrategias de intervención a nivel cognitivo e interpersonal se presentan como poco fructíferas para el cambio de tal contenido.

En este sentido, la producción de leyes que prohíben la discriminación, y la construcción de políticas públicas de enfrentamiento y combate a las desigualdades sociales y raciales, como cuotas sociales para grupos minoritarios, se presentan como las intervenciones de mejor pronóstico social en la resolución del problema del prejuicio racial en Brasil.

\section{Referencias}

Abric, J. C. (2003). Abordagem estrutural das representações sociais: desenvolvimentos recentes. En P. H. F. Campos \& M. C. da S. Loureiro. (Eds.), Representações sociais e práticas educativas (pp. 37-57). Goiânia: UCG.

Allport, G. (1979). The nature of prejudice. New York: Addison-Wesley Publishing Company.

Amodio, D. M., \& Devine, P. G. (2006). Stereotyping and evaluation in implicit race bias: evidence for independent constructs and unique effects on behavior. Journal of Persona- lity and Social Psychology,91(4), 652-661. Doi: 10.1037/0022-3514.91.4.652

Batista, J. R. M., Leite, E. L., Camino, L., \& Torres, A. R. R. (2014). Negros e nordestinos: similaridades nos estereótipos raciais e regionais. Psicologia Política, 14(30), 325-345.

Bergsieker, H. B., \& Leslie, L. M. (2012). Stereotyping by omission: eliminate the negative, accentuate the positive. Journal of Personality and Social Psychology, 102(6), 1214-1238. Doi: 10.1037/a0027717

Binggeli, S., Krings, R., \& Sczesny, S. (2014). Stereotype content associated with immigrant groups in Switzerland. Swiss Journal of Psychology, 73(3), 123-133. Doi: 10.1024/1421-0185/a 000133

Brewer, M. B. (1994). The psychology of prejudice: getting it all together. En M. Zanna \& J. Olson (Org.), The psychology of prejudice: The Ontario symposium on personality and social psychology (p. 371). Hillsdale, NJ: Erlbaum.

Brown, R. J. (2010). Prejudice: its social psychology. Massachusetts: Blackwell Publishers Inc.

Cabecinhas, R. (2004). Estereótipos sociais, processos cognitivos e normas sociais. Comunicación oral presentada en el seminario Cultural Diversity on The Midia: Identity, Stereotypes and Ethnocentrism em Amsterdã, Holanda el18 de mayo de 2004. Recuperado de https://repositorium.sdum.uminho.pt/bitstream/1822/39422/1/ RC_estereotipos_sociais.pdf

Camino, L., da Silva, P., Machado, A., \& Pereira, C. (2001). A face oculta do racismo no brasil: uma análise psicossociológica. Revista de Psicologia Política, 1(1), 13-36.

Camino, L., Álvaro, J. L., Torres, A. R. R., Garrido, A., Morais, T. Barbosa, J. (2013a). explaining social discrimination: racism in brazil and xenophobia in Spain, Spanish Journal of Psychology, 16(73), 1-13. Doi: 10.1017/sjp.2013.65

Camino, L., Gouveia, M. L., Maia, L., Paz, M. M. A., \& Santos, M. L. O. (2013b). Repertórios discursivos contemporâneos sobre as desigualdades 
raciais no Brasil: um estudo com estudantes paraibanos de pós-graduação. Psicologia e Sociedade (Impresso), 25, 113-122. Doi: 10.1590/ S0102-71822013000100013 Camargo, B. V., \& Justo, A. M. (2013). Iramuteq: um software gratuito para análise de dados textuais. Temas em Psicologia, 21(2), 513-518. Doi: 10.9788/ TP2013.2-16

Carvalho, N. C., Lima, M. E. O., Faro, A., \& Silva, C. A. F. (2012). Representações sociais dos ciganos em Sergipe: contato e estereótipos. Psicologia e Saber Social, 1(2), 232-244.

Carvalho, R. A. \& Rocha, S. P. (2016). As mulheres negras em movimento no Brasil: atuação política da Bamidelê - Organização de Mulheres Negras na Paraíba. Gênero, 16(2), 71-89. Doi: 10.22409/rg.v16i2.802

Crandall, C. S., Bahns, A. J., Warner, R., \& Schaller, M. (2011). Stereotypes as justifications of prejudice. Personality and Social Psychology Bulletin, 37(11), 1488-1498. Doi: 10.1177/0146167211411723

Doise, W. (1986). Levels of explanation in social psychology. Cambridge: Cambridge University Press.

Fehr, J., Sassenberg, K., \& Jonas, K. J. (2012). Willful stereotype control the impact of internal motivation to respond without prejudice on the regulation of activated stereotypes. Zeitschrift für Psychologie , 220(3),180-186. Doi: 10.1027/2151-2604/a000111

Fiske, S. T \& Taylor, S. E. (1991). Social cognition. New York: McGraw-Hill.

Fiske, S. T. Cuddy, A. J. C., Glick, P., \& Xu, J. (2002). A model of (often mixed) stereotype content: competence and warmth respectively follow from perceived status and competition. Journal of personality and social psychology, 82(6), 878-902. Doi: 10.1037//0022-3514.82.6.878

Fiske, S. T., Cuddy, A. J. C., \& Glick, P. (2007). Universal dimensions of social cognition: warmth and competence. Trends in Cognitive Sciences,
11(2), 77-83. Doi: https://doi.org/10.1016/j. tics.2006.11.005

Fiske, S. T., Xu, J., Cuddy, A. C., \& Glick, P. (1999). (Dis)respecting versus (dis)liking: Status and interdependence predict ambivalent stereotypes of competence and warmth. Journal of Social Issues, 55(3), 473-489.

Freyre, G. (1933/1998). Casa-Grande \& Senzala. Río de Janeiro: Editora Record.

Gaertner, S. L., \& Dovidio, J. F. (1986). The aversive form of racism. En J. F. Dovidio, \& S. L. Gaertner(Orgs.), Prejudice, discrimination, and racism (pp. 61-89). Orlando, Florida: Academic.

Gawronski, B., Deutsch, R. Mbirkou, S., Seibt, B., \& Strack, F. (2008). When "Just say no" is not enough: Affirmation versus negation training and the reduction of automatic stereotype activation. Journal of Experimental Social Psychology, 44, 370-377. Doi: 10.1016/j. jesp.2006.12.004

Gilbert, G. M. (1951). Stereotype persistence and change among college students. Journal of $A b$ normal and Social Psychology, 46, 245-254. Doi: http://dx.doi.org/10.1037/h0053696

Gomes Lino, N. (2002). Trajetórias escolares, corpo negro e cabelo crespo: reprodução de estereótipos ou ressignificação cultural? Revista Brasileira de Educação, 21, 40-51. Doi: http://dx.doi. org/10.1590/S1413-24782002000300004.

Gordijn, E. H., Koomer, W., \& Stapel, D. A. (2001). Level of prejudice in relation to knowledge of cultural stereotypes. Journal of Experimental Social Psychology, 37(2), 150-157. Doi: 10.1006/jesp.2000.1443

Guan, Y., Deng, H., \& Bond, M. H. (2010). Examining stereotype content model in a Chinese context: inter-group structural relations and Mainland Chinese's stereotypes towards Hong Kong Chinese. International Journal of Intercultural Relations, 34, 393-399. Doi:10.1016/j. ijintrel.2010.04.003

IBGE. (2014). Síntese dos indicadores sociais: uma análise das condições de vida da população 
brasileira. Rio de Janeiro: Instituto Brasileiro de Geografia e Estatística.

Jones, J. M. (1997). Prejudice and racism. New York: McGraw-Hill.

Karlins, M., Coffman, T. L., \& Walters, G. (1969). On the fading of social stereotypes: studies in three generations of college students. Journal of Personality and Social Psychology, 13, 1-16. Doi: http://dx.doi.org/10.1037/h0027994

Katz, D., \& Braly, K. (1933). Verbal stereotypes and racial prejudice. Journal of Abnormal and Social Psychology, 133(2), 280-290.

Katz, I., \& Hass, R. G. (1988). Racial ambivalence and American value conflict: correlational and priming studies of dual cognitive structures. Journal of Personality and Social Psychology, 55, 893-905.

Kinder, D. R., \& Sears, D. O. (1981). Prejudice and politics: symbolic racism versus racial threats to the good life. Journal of Personality and Social Psychology, 40, 414-431. Doi: 0022-3514/81 /4003-0414S00.75

Krüger, H. (2004). Cognição, estereótipos e preconceitos sociais. En M. E. O. Lima \& M. E. Pereira (Orgs.). Estereótipos, preconceitos e discriminação: Perspectivas teóricas e metodológicas (pp. 23-40). Salvador: EDUFBA.

Lima, M. E. O., \& Vala, J. (2004). As novas formas de expressão do preconceito e do racismo. Estudos de psicologia (Natal), 9(3), 401-411. Doi: http://dx.doi.org/10.1590/S1413294X2004000300002

Moreno, S. (2016). Cidade mais negra fora da África, Salvador completa 467 anos. Recuperado de http://agenciabrasil.ebc.com.br/geral/noticia/2016-03/os-467-anos-de-salvador-cidademais-negra-fora-da-africa

Munanga, K. (2007). Políticas de ação afirmativa em benefício da população negra no Brasil: um ponto de vista em defesa de cotas. Sociedade e Cultura, 4(2), 31-42. Doi: https://doi. org/10.5216/sec.v4i2.515
Oakes, P. J., Haslam, S. A., \& Turner, J. C. (1994). Stereotyping and social reality. Oxford: Blackwell.

Oliveira, C. L. P. \& Barreto, P. C. S. (2003). Percepção do racismo no Rio de Janeiro. Estudos Afro-Asiáticos, 25(2), 183-213. Doi: http://dx. doi.org/10.1590/S0101-546X2003000200001

Paixão, M., Rossetto, I., Montovanele, F., \& Carvano, L. M. (Orgs.). (2010). Relatório anual das desigualdades raciais no Brasil; 2009-2010. Recuperado de http://www.palmares.gov.br/ wp-content/uploads/2011/09/desigualdades_raciais_2009-2010.pdf

Pedersen, A., \& Walker, I. (1997). Prejudice against Australian aborígines: old-fashioned and modern forms. European Journal of Social Psychology, 27, 561-587. Doi: 10.1002/(SICI)1 099-0992(199709/10)27:5<561::AID-EJSP833>3.0.CO;2-3

Pereira, M. E. (2015). Enfrentando preconceitos e estereótipos. Na escola, no trabalho, nas ruas e os que sobrevivem em cada um de nós. Recuperado de http://estereotipos.net/

Pereira, C., Torres, A. R. R., \& Almeida, S. T. (2003). Um estudo do preconceito na perspectiva das representações sociais: análise da influência de um discurso justificador da discriminação no preconceito racial. Psicologia: Reflexão e Crítica, 16(1), 95-107. Doi: doi.org/10.1590/ S0102-79722003000100010.

Pereira, C. R., \& Vala, J. (2010). Do preconceito à discriminação justificada. In-Mind Português, 1(2-3), 1-13. Recuperado de http://hdl.handle. net $/ 10451 / 893$

Pettigrew, T. F., \& Meertens, R. W. (1995). Subtle and blatant prejudice in western Europe. European Journal of Social Psychology, 25, 203-226. Doi: 10.1002/ejsp.2420250106

Ratinaud, P. (2009). Iramuteq: interface de R pour les analyses multidimensionnelles de textes et de questionnaires [computer software]. Recuperado de http://www.iramuteq.org 
Rocha, A. A. (2010). Representações Sociais do jogo de regras: um estudo entre professores de educação física da rede pública estadual da Bahia (tesis de posgrado, Universidade federal da Bahía, Bahía, Brasil).

Sadler, M. S., Meagor, E. L., \& Kaye, K. E. (2012). Stereotypes of mental disorders differ in competence and warmth. Social Science \& Medicine, 74, 915-922. Doi:10.1016/j.socscimed.2011.12.019

Schwarcz, L.M. \& Starling, H. M. (2015). Brasil: uma biografia. São Paulo: Companhia das letras.

Schucman, L. V. (2014). Sim, nós somos racistas: estudos psicossocial da branquitude paulistana. Psicologia e Sociedade, 26(1), 8394. Doi: http://dx.doi.org/10.1590/S010271822014000100010

Sherman, J. W., Stroessner, S. J., Conrey, F. R., \& Azam, O A. (2005). Prejudice and stereotype maintenance processes: attention, attribution, and individuation. Journal of Personality and Social Psychology, 89(4), 607-622. Doi: 10.1037/0022-3514.89.4.607

Sparkman, D. J., \& Eidelman, S. (2016). Putting myself in their shoes: ethnic perspective taking explains liberal-conservative differences in prejudice and stereotyping. Personality and Individual Differences, 98, 1-5. Doi: https://doi. org/10.1016/j.paid.2016.03.095

Tajfel, H. (1981). Grupos humanos e categorias sociais I. Lisboa: Livros Horizonte.

Tajfel, H., \& Turner, J. (1979). An integrative theory of intergroup conflict. En W.G Austin \& S.
Worchel (Eds). The Social Psychology of Intergroup Relations (pp. 33-47). Monterey: CA: Brooks/Cole.

Techio, E. M. (2011). Estereótipos sociais como preditores das relações intergrupais. Em E. M.Techio, \& M. E. O. Lima (Eds.), Cultura e produção das diferenças: estereótipos e preconceitos no Brasil, Espanha e Portugal (pp. 22-36). Brasília: Technopolitik.

Techio, E. M., Gonçalves, J. P., \& Costa, P. N. (2016). Representação social da sustentabilidade na construção civil: a visão de estudantes universitários. Ambiente \& Sociedade, 19(2), 187-204. Doi: 10.1590/1809-4422ASOC130991V1922016

Turra, C., \& Venturi, G. (1995). Racismo cordial: a mais completa análise sobre preconceito de cor no Brasil. São Paulo: Ática.

Unnever, J. D., \& Cullen, F. T. (2012). White perceptions of whether African Americans and Hispanics are prone to violence and support for the death penalty. Journal of Research in Crime and Delinquency, 49(4), 519-544. Doi: $10.1177 / 0022427811415533$

Werneck, J. (2016). Racismo institucional e saúde da população negra. Saúde Soc. São Paulo, 25(3), 535-549. Doi: 10.1590/S0104-129020162610 Waiselfisz, J. J, (2016). Mapa da violência: homicídios por arma de fogo no Brasil. Recuperado de www.mapadaviolencia.org.br/pdf2016/Mapa2016_armas_web.pdf

\section{Recibido: 05 de octubre de 2017 Aprobado: 09 de octubre de 2018}

